\title{
Does the genetic and familial background of males undertaking ICSI affect the outcome?
}

\author{
M. Maiburg • B. Alizadeh • P. Kastrop • M. Lock • \\ S. Lans • J. Giltay
}

Received: 8 January 2009/Accepted: 18 May 2009/Published online: 23 June 2009

(C) The Author(s) 2009. This article is published with open access at Springerlink.com

\begin{abstract}
Purpose To investigate whether the success rate of ICSI is (1) related to the etiology of infertility or (2) adversely affected by a family history of potential genetic disorders. Methods All men with an ICSI indication in our hospital between 1994 and 2005 were included in our cohort study. Data on the ICSI process, etiology of infertility, and family history were collected. ICSI success rates of infertility subgroups and a subgroup with a positive family history were compared to a group with unknown etiology and a negative family history.

Results There was no significant difference in clinical pregnancy or delivery rates between the subgroups. Couples achieving a pregnancy underwent significantly more ICSI cycles compared to couples not achieving a pregnancy.
\end{abstract}

Capsule ICSI success rate is neither related to the cause of infertility nor adversely affected by a family history positive for potential genetic disorders.

M. Maiburg $\cdot$ B. Alizadeh $\cdot$ S. Lans $\cdot$ J. Giltay

Department of Medical Genetics,

University Medical Center Utrecht,

Utrecht, The Netherlands

P. Kastrop

Department of Reproductive Medicine,

University Medical Center Utrecht,

Utrecht, The Netherlands

M. Lock

Department of Urology, University Medical Center Utrecht, Utrecht, The Netherlands

M. Maiburg $(\square)$

Department of Medical Genetics,

University Medical Center Utrecht,

P.O. Box 85090, 3508 AB Utrecht, The Netherlands

e-mail: m.maiburg@umcutrecht.nl
Conclusion Our results suggest that the success rate of ICSI treatment is not related to the cause of infertility or a family history positive for potential genetic disorders.

Keywords Etiology · Family history · Intracytoplasmic sperm injection · Male infertility Success rate

\section{Introduction}

Since its introduction in 1991, intracytoplasmic sperm injection (ICSI) has been a treatment option for couples with male infertility. Worldwide it has an average pregnancy success rate of $25-30 \%$ of pregnancies per treatment cycle. This figure is equal to that of in vitro fertilization (IVF) [1-3]. In recent years the success rate of ICSI has not substantially improved, despite new procedures such as preimplantation genetic screening (PGS) [3-5]. Several studies have tried to identify the underlying causes of the limited success rate of ICSI, for example advanced maternal age or sperm origin (testicular, epididymal or ejaculated). However, no good explanation has been found yet [6-8].

On the basis of the etiology of in-/subfertility, different subgroups of ICSI candidates can be distinguished: obstructive azoospermia, including congenital uni- or bilateral agenesis of the vas deferens (CUAVD or CBAVD) caused by mutations in the cystic fibrosis transmembrane conductance regulator (CFTR) gene, and non-obstructive in-/subfertility, which in a number of cases is caused by either chromosomal abnormalities or Y-deletions [9, 10].

Another possible indicator of the ICSI success rate might be found in the family history of couples with in-/ subfertility. A family history of congenital anomalies or serious disorders might be, at least in part, genetically determined and therefore reflect to some extent a decreased 
viability of embryos/offspring in infertile couples. It is possible that physiological or anatomical abnormalities determine the fate of the fetus and modify the success rate of ICSI.

We hypothesized that the success of ICSI treatment could be related to the cause of infertility. We first aimed to determine the prevalence of different etiological subgroups in a large historical cohort of 1565 ICSI couples during the first 11 years of the use of ICSI in our hospital. Secondly, we investigated whether the success rate of ICSI was (1) related to the etiology of infertility or (2) adversely affected by a family history of potential genetic disorders.

\section{Methods}

Study population

ICSI treatment was introduced in the University Hospital in Utrecht, the Netherlands, in 1994. From then to 2005 all men with an indication for ICSI treatment were included in our study ( $n=1565$, of which 1487 had a genetic follow up, see flowchart in Fig. 1). Indications for ICSI were severe oligoasthenoteratozoospermia (OAT) [11] or total fertilization failure in at least two previous in vitro fertilization cycles. This means that males with normospermia could also be included. There was a national moratorium in the Netherlands on using surgically retrieved sperm for ICSI during part of the study period (1996-2001), so that ejaculated spermatozoa were used in the majority of cases, explaining why a relatively low number of men with obstructive azoospermia, such as CBAVD, were included in this ICSI group. For those patients with obstructive azoospermia, spermatozoa were surgically retrieved by microsurgical epididymal sperm aspiration (MESA) or percutaneous epididymal sperm aspiration (PESA).

\section{Data collection}

We performed a historical cohort study, obtaining data from the medical records of ICSI candidates. As part of the ICSI procedure, karyotyping was performed in all the males. Soon after the introduction of ICSI, Y chromosome deletion analysis was also introduced in the diagnostic

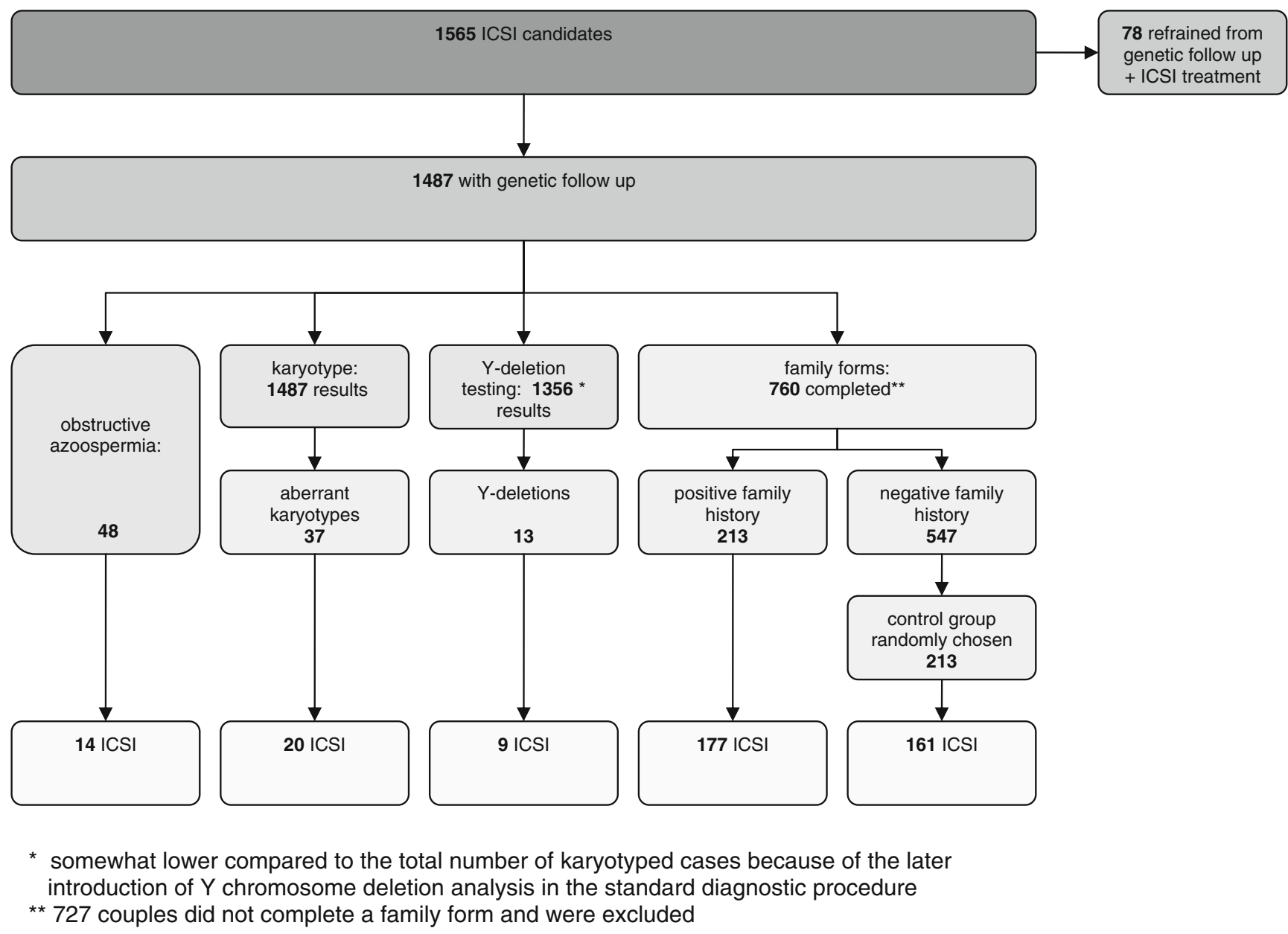

Fig. 1 Flowchart of study 
procedure (explaining the somewhat lower number of total cases in which $\mathrm{Y}$ chromosome deletion analysis was performed compared to the total number of karyotyped cases). In case of CBAVD or obstructive azoospermia, CFTR mutation analysis was performed. Couples were also asked to complete a family history form on the occurrence of 31 disorders in first-, second-, and third-degree relatives. These disorders were classified into seven categories: (1) spontaneous abortions, (2) stillborn children or children who had died in the early postpartum period, (3) congenital malformations, (4) mental retardation (not otherwise specified), (5) in-/subfertility, (6) multifactorial and/or late-onset diseases (e.g., cardiovascular disease, cancer, epilepsy), and (7) others (including consanguinity between partners). A family history was considered aberrant (positive) if any disorder in the above categories was reported. Couples with a positive family history were informed about the possible genetic risk by a clinical geneticist.

We recorded the number of treatment cycles, embryonic transfers, and pregnancies for all couples. If a pregnancy was achieved, we also documented the course (ongoing pregnancies, abortions or stillbirths) and outcome (live born children) of the pregnancies, as well as the number of multiple pregnancies. Although congenital malformation data were not systematically recorded, information on serious congenital malformations was available in a few cases. In July 2008 (3 years after the initial data collection), the follow up data were collected.

\section{Data analysis}

ICSI couples were divided into the following subgroups: (1) aberrant karyotyping, (2) Y-deletion, (3) obstructive azoospermia, and (4) positive family history. From the remaining couples without a positive family history (i.e., idiopathic infertility without any abnormalities recorded in our subgroups), we chose a number of ICSI couples with idiopathic infertility at random to serve as an internal control group; this subgroup was the same size as the positive family history group (Fig. 1).

The course of the ICSI treatment for the different subgroups was recorded. We compared: (1) success in terms of clinical pregnancy rate (number of pregnancies / number of ICSI cycles), (2) success in terms of delivery rate (number of pregnancies-number of abortions / number of ICSI cycles), and (3) number of couples achieving at least one clinical pregnancy or live born child ("success couples"). Individual subgroups were compared to (1) the internal control group and (2) the total of the remaining subgroups.

The success rates were treated as numerical variables. Statistical analysis was performed using the Mann-Whitney or Chi-squared test. Data were examined for normality using the Komorono-Smironof test, and a linear regression model for success rate was fitted with multiple causes of infertility.

\section{Results}

Prevalence of etiological factors of infertility

The prevalence of the causes of infertility and positive family history in our study population is shown in Table 1. The most prevalent cause of infertility was obstructive azoospermia (3.2\%) followed by an aberrant male karyotype (2.5\%) and Y-deletions (0.95\%). The azoospermia subgroup $(n=48)$ included CBAVD $(n=41)$, CUAVD $(n=1)$, and other forms of obstructive azoospermia $(n=6)$. A CFTR mutation was found in 27 cases (56\%): 14 patients were heterozygous for a CFTR mutation $(29 \%)$, one was homozygous $(2 \%)$, and 12 were compound heterozygous (25\%). In the aberrant karyotyping subgroup, Robertsonian translocations (27\%) and sex chromosomal aberrations (22\%) were the most common chromosomal abnormality. In the Y-deletion subgroup, a deletion of either AZFc $(11 ; 85 \%), A Z F b+c(1 ; 8 \%)$, or AZFa $(1 ; 8 \%)$ was found.

There were 760 family forms available, of which 213 (28\%) revealed a positive family history for one (or more) of the disorders listed in Table 1. Three couples in the aberrant karyotyping group, three couples in the obstructive azoospermia group, but none in the Y-deletion group, also had a positive family history.

\section{Success rates}

The course of ICSI treatment in the total cohort and the different subgroups is shown in Table 2. In total, 1088 ICSI cycles were performed in 381 candidates, yielding 361 pregnancies and 331 live born children. The overall delivery rate ( $\geq 1$ child born / ICSI cycle) was $27 \%$ (295/ 1088). The success rates and 'success couples' for the different subgroups are given in Table 3.

The clinical pregnancy rate and delivery rate for couples with idiopathic male infertility (i.e., the internal control group) was $33 \%$ and $25 \%$ respectively (Table 3b). The clinical pregnancy rates of the other categories were $41 \%$ (obstructive azoospermia, $p=0.33$ ), $37 \%$ (aberrant karyotype, $p=0.51$ ), 38\% (Y-deletion, $p=$ 0.55 ), and $33 \%$ (positive family history, $p=0.99$ ) respectively. Compared to the control group there was no significant difference in delivery rate for patients with obstructive azoospermia $(41 \%, p=0.50)$, patients with an aberrant male karyotype $(35 \%, p=0.13)$, patients with a Y-deletion $(34 \%, p=0.28)$, or patients with a positive 
Table 1 Prevalence of the causes of infertility in our study population $(n=1487)$

\begin{tabular}{|c|c|c|c|c|c|c|c|}
\hline \multicolumn{6}{|l|}{ Causal } & \multicolumn{2}{|l|}{ Associated } \\
\hline \multicolumn{2}{|c|}{ Obstructive azoospermia ${ }^{a}$} & \multicolumn{2}{|l|}{ Aberrant karyotype } & \multicolumn{2}{|c|}{ Y-deletion } & \multicolumn{2}{|l|}{ Positive family history ${ }^{\mathrm{b}}$} \\
\hline \multicolumn{2}{|l|}{$48 / 1487$} & \multicolumn{2}{|l|}{$37 / 1487$} & \multicolumn{2}{|l|}{$13 / 1365$} & \multicolumn{2}{|l|}{$213 / 760$} \\
\hline \multicolumn{2}{|l|}{$(3.2 \%)^{\mathrm{c}}$} & \multicolumn{2}{|l|}{$(2.5 \%)$} & \multicolumn{2}{|l|}{$(0.95 \%)$} & \multicolumn{2}{|l|}{$(28.0 \%)$} \\
\hline CFTR mutations & $27(56 \%)$ & Robertsonian translocations & $10(27 \%)$ & $\mathrm{AZFa}$ & $1(8 \%)$ & (1) spontaneous abortions & $40(19 \%)$ \\
\hline - heterozygous & $14(29 \%)$ & sex chromosomal abnormalities & $8(22 \%)$ & $\mathrm{AZFb}$ & $0(0 \%)$ & (2) stillborn or postpartum (dead) & $7(3 \%)$ \\
\hline - homozygous & $1(2 \%)$ & reciprocal translocations & $7(19 \%)$ & $\mathrm{AZFc}$ & $11(85 \%)$ & (3) congenital malformations ${ }^{\mathrm{d}}$ & $37(17 \%)$ \\
\hline \multirow{4}{*}{$\begin{array}{l}\text { - compound } \\
\text { heterozygous }\end{array}$} & $12(25 \%)$ & mozaisicm & $6(16 \%)$ & $\mathrm{AZFb}+\mathrm{c}$ & $1(8 \%)$ & (4) mental retardation ${ }^{\mathrm{e}}$ & $24(11 \%)$ \\
\hline & & marker chromosomes & $4(11 \%)$ & & & (5) in-/subfertility & $6(3 \%)$ \\
\hline & & inversions & $2(5 \%)$ & & & (6) multifactorial / late onset ${ }^{\mathrm{f}}$ & $48(23 \%)$ \\
\hline & & & & & & (7) other ${ }^{g}$ & $54(25 \%)$ \\
\hline
\end{tabular}

${ }^{\text {a }}$ subgroup includes CBAVD $(n=41)$, CUAVD $(n=1)$ and other forms of obstructive azoospermia $(n=6)$

${ }^{\mathrm{b}}$ more than one item can be positive in one familiy

${ }^{\mathrm{c}}$ relatively low prevalence in this cohort due to the Dutch moratorium on using surgically retrieved sperm

${ }^{\mathrm{d}}$ including club feet, cleft lip/palate, congenital heart malformations, hydrocephaly and neural tube defects

${ }^{\mathrm{e}}$ including Down syndrome

${ }^{f}$ including cardiovascular disease, diabetes mellitus, cancer, epilepsy, asthma/bronchitis and allergies

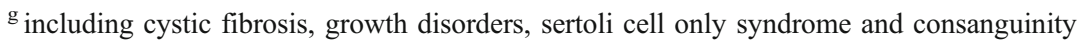

family history $(27 \%, p=0.70)$. There was also no significant difference in pregnancy- or delivery rate when comparing the individual subgroups to the rest of the cohort (data not shown).

Of the 161 couples with idiopathic infertility (i.e. the internal control group), 102 (63\%) achieved at least one pregnancy (Table 3c), compared to 10 in the obstructive azoospermia subgroup $(71 \%, p=0.54), 14$ in the aberrant karyotype subgroup $(70 \%, p=0.56)$, seven in the Y-deletion subgroup $(78 \%, p=0.38)$ and 125 of the positive family history subgroup $(71 \%, p=0.16)$.

Of the 161 couples of the control group, 89 (55\%) had at least one child after ICSI treatment, compared to ten in the obstructive azoospermia subgroup $(71 \%, p=0.24)$, 14 in the aberrant karyotype subgroup $(70 \%, p=0.21)$, seven in the Y-deletion subgroup $(78 \%, p=0.19)$, and 112 of the positive family history subgroup $(63 \%, p=0.13)$.

\section{Discussion}

A large cohort of 1565 ICSI couples was analyzed with respect to the genetic causes of male infertility. We analyzed the relationship between the causes of infertility or a positive family history for potential genetic disorders and ICSI success rates. We found no significant differences for any of these subgroups with respect to the clinical pregnancy and delivery rates. Many studies have looked at factors influencing the outcome of ICSI [6-8], however, to our knowledge, this is the first in which the possible adverse effect of a positive family history was included.

Distribution of etiological subgroups

The rate we determined for male chromosome abnormalities $(2.5 \%)$ was somewhat lower than those reported in

Table 2 Course of ICSI treatment

\begin{tabular}{lcccrrr}
\hline & Control group & Obstructive azoospermia & Aberrant karyotype & Y-deletion & Positive family history & Total \\
\hline $\begin{array}{l}\text { Couples starting ICSI } \\
\text { in our centre, } \mathrm{n}\end{array}$ & 161 & 14 & 20 & 9 & 177 & 381 \\
ICSI cycles, n & 448 & 29 & 54 & 29 & 528 & 1088 \\
Embryonic transfers, n & 396 & 24 & 48 & 25 & 462 & 172 \\
Clinical pregnancies, n & 146 & 12 & 20 & 11 & 15 & 365 \\
Multiple pregnancies, n & 17 & 3 & 1 & 0 & 36 \\
Abortions, n & 32 & 0 & 20 & 10 & 155 \\
Live born children, n & 131 & 15 & & 331 \\
\hline
\end{tabular}


Table 3 Outcomes of ICSI treatment

\begin{tabular}{|c|c|c|c|c|c|}
\hline & Control group & Obstructive azoospermia & Aberrant karyotype & Y-deletion & Positive family history \\
\hline \multicolumn{6}{|l|}{ a) Couples and cycles } \\
\hline $\begin{array}{l}\text { Couples starting ICSI in } \\
\text { our centre, } \mathrm{n}\end{array}$ & 161 & 14 & 20 & 9 & 177 \\
\hline $\begin{array}{l}\text { Median number of cycles } \\
\text { per couple (range) } \\
\text { b) Success rates }\end{array}$ & $2.34(1-10)$ & $1.92(1-5)$ & $2.26(1-8)$ & $3.02(1-6)$ & $2.66(1-10)$ \\
\hline $\begin{array}{l}\text { Clinical pregnancies / ICSI } \\
\text { cycles }(\%)\end{array}$ & 33 & 41 & 37 & 38 & 33 \\
\hline Relative success $(95 \% \mathrm{CI})$ & - & $1.24(0.63-2.41)$ & $1.12(0.60-2.06)$ & $1.15(0.48-2.72)$ & $1.00(0.73-1.35)$ \\
\hline $\begin{array}{l}\text { Deliveries } \geq 1 \text { child / ICSI } \\
\text { cycles }(\%)\end{array}$ & 25 & 41 & 35 & 34 & 27 \\
\hline Relative success $(95 \% \mathrm{CI})$ & - & $1.64(0.82-3.24)$ & $1.4(0.72-2.69)$ & $1.36(0.52-3.51)$ & $1.08(0.75-1.54)$ \\
\hline \multicolumn{6}{|l|}{ c) (Un)successful couples } \\
\hline $\begin{array}{l}\text { Couples achieving at least } 1 \\
\text { pregnancy, } \mathrm{n}(\%)^{*}\end{array}$ & $102(63)$ & $10(71)$ & $14(70)$ & $7(78)$ & $125(71)$ \\
\hline $\begin{array}{l}\text { Median number of cycles } \\
\text { per couple (range) }\end{array}$ & $2.52(1-10)$ & $1.89(1-5)$ & $2.77(1-8)$ & $3.43(1-6)$ & $2.72(1-10)$ \\
\hline $\begin{array}{l}\text { Couples not achieving } \\
\text { a pregnancy, } \mathrm{n}(\%)\end{array}$ & $59(37)$ & $4(29)$ & $6(30)$ & $2(22)$ & $52(29)$ \\
\hline $\begin{array}{l}\text { Median number of cycles } \\
\text { per couple (range) }\end{array}$ & $2.11(1-8)$ & $2.00(1-3)$ & $1.80(1-3)$ & $2.50(2-3)$ & $2.19(1-6)$ \\
\hline $\begin{array}{l}\text { Couples having at least on } \\
\text { child after ICSI }\end{array}$ & $89(55)$ & $10(71)$ & $14(70)$ & $7(78)$ & $112(63)$ \\
\hline $\begin{array}{l}\text { Median number of cycles } \\
\text { per couple (range) }\end{array}$ & $2.52(1-10)$ & $1.89(1-5)$ & $2.77(1-8)$ & $3.33(1-6)$ & $2.56(1-9)$ \\
\hline $\begin{array}{l}\text { Couples without children } \\
\text { after ICSI }\end{array}$ & $72(45)$ & $4(29)$ & $6(30)$ & $2(22)$ & 65 (37) \\
\hline $\begin{array}{l}\text { Median number of cycles } \\
\text { per couple (range) }\end{array}$ & $2.16(1-8)$ & $2.00(1-3)$ & $1.80(1-3)$ & $2.50(2-3)$ & $2.79(1-10)$ \\
\hline
\end{tabular}

$*=$ positive predictive value (PPV)

previous studies, which range from 3.5 to $7.7 \%$ for the overall ICSI group $[9,12,13]$. This could be explained by the fact that our study included mainly males with ejaculated sperm due to a Dutch moratorium on using surgically retrieved sperm for ICSI during a major part of the study period. Therefore males with idiopathic azoospermia (in whom the highest rates of chromosome abnormalities can be found), were not included in this study. Moreover, males with obstructive azoospermia were documented as a separate group. In addition, one of the indications for ICSI treatment was "total fertilization failure in at least two previous IVF cycles", thus including males with normospermia as well. Compared to the published rates of chromosome abnormalities in males with OAT, our figures do not differ much from those of Bor et al. (2.7\%) [12], Tuerlings et al. (3.5\%) [13], and Vincent et al. $(0.5 \%$ in mild and $4.3 \%$ in moderate OAT) [14].

The rate of Y-chromosome deletions in the DAZ region $(0.95 \%)$ in our study population was comparable to that reported by Bor et al. (0.75\% in an overall ICSI group) [12] and somewhat lower compared to that by Cruger et al. (2\% in a severe OAT group) [15].

Our cohort of patients in whom surgically obtained sperm were used consisted of patients with CBAVD ( $n=$ 41), CUAVD $(n=1)$ and other forms of obstructive azoospermia $(n=6)$. The number of CFTR mutations found in the latter group is usually smaller than that in the CBAVD group [16]. The number of cases with double allelic $(13 / 48=27 \%)$ or monoallelic $(14 / 48=29 \%)$ mutations in the CFTR gene in our obstructive azoospermia group seems to be lower than in previously published reports, especially with respect to the double allelic mutations. Mak et al. [16] reported two mutations in $40 \%$ of CBAVD patients and $25 \%$ of obstructive azoospermia patients (CBAVD, CUAVD and idiopathic obstruction), and Claustres et al. [17] reported two mutations in $47 \%$ of CBAVD patients). However, the numbers of mutations reported in different publications show a wide variation. We do not have a clear explanation for this discrepancy. Overall, at least with respect to the small subset of genetic 
aberrations identified in this study, our ICSI cohort is similar to the other published ICSI cohorts.

With respect to the self-reporting of family history, we realize that not everyone might have been equally accurate in reporting disorders in family members. Couples who are concerned about the risks for their progeny may report more extensively than couples who are less concerned about this risk, or whose only concern is to have children after ICSI irrespective of any burden of familial disorders. We cannot exclude that some couples in the negative family history group were in fact non-reporters of a positive family history and they should not therefore have been included in the control group. Furthermore we are well aware that we did not discriminate between the different categories of reported disorders and the relationship of the affected family member (more distant or close) on the one hand and the ICSI outcome on the other hand. Therefore it cannot be excluded that more serious disorders in close family members have an adverse effect on ICSI outcome, whereas milder disorders in more distant family members may not.

\section{ICSI success rates}

We compared the ICSI success rates of the different etiological subgroups, including the positive family history subgroup, to an internal control group (negative family history). The rates for each of these subgroups were similar or higher compared to the previously reported success rate of ICSI treatment (25-30\%) [1-3], ranging from 33-41\% for the clinical pregnancy rate and $25-41 \%$ for the delivery rate.

Interestingly, when comparing ICSI candidates with a known etiology (i.e., either aberrant karyotype, Y-deletion, or obstructive azoospermia) to the positive family history group or control group, the last two groups turn out to have the lowest success rates in this study. Although this difference was not significant, we cannot exclude that unknown genetic factors could, at least in part, explain the infertility in these groups and influence the ICSI success rates. On the other hand, the relatively high success rate in the obstructive azoospermia group might be explained by their essentially normal sperm production and that the reason they need help is only due to a physical circumstance, such as obstruction.

The different subgroups were comparable with regard to the mean number of cycles, number of multiple pregnancies, and number of abortions. However, there was a significant difference $(p=0.013)$ between the mean number of cycles in the overall group of couples achieving at least one pregnancy compared to couples not achieving a pregnancy (Table 3c). Accordingly, more ICSI cycles lead to more couples having at least one child after ICSI in the overall group, although the latter observation was not significant $(p=0.192)$. For clinical practice, this means that couples could be counselled towards undergoing more cycles, expecting that success can still be achieved regardless of the underlying aetiology. This was to be expected for the control group. However, we showed that also for the other subgroups, with the exception of the obstructive azoospermia group that already has a relatively high success rate, it is a low number of cycles rather than the etiology which is the cause of the low success rate.

One of the limitations in this study was that the overall sizes of the subgroups were relatively small, although this was a reasonably sized cohort of total ICSI candidates. After intake and diagnostic investigations, a number of candidates did not have ICSI treatment in our hospital (143/ $524=27 \%$ ). During the study period, waiting lists in different hospitals varied considerably, leading couples to 'shop around'. Some couples went abroad for ICSI treatment because of the Dutch moratorium (see Methods). Other couples opted for adoption, artificial insemination, or IVF with donor sperm, or refrained from treatment because of emotional burden. Previous studies have already shown that $44 \%$ of males with a chromosomal aberration [18] and $21 \%$ of males with a Y-deletion [19] refrained from ICSI treatment.

Despite the limitations of this study, our data from one large cohort are interesting since we could compare the success of ICSI treatment in different subgroups of in-/ subfertility, including one that has not been previously studied, i.e., with a positive family history. We found no significant relationship between the ICSI success rate and the causes of male infertility. Probably evaluating the various population-, cyto-, and molecular genetic features may be of significance for counselling but they appear to be of no value for prediction of the effectiveness of ICSI. However, our subgroups were relatively small, and further analysis in larger subgroups might yield different results. It remains unclear whether the ICSI success rate is limited by unknown factors or by factors inherent to the technique.

\section{Conclusions}

The success rate of ICSI treatment is neither related to the cause of infertility nor adversely affected by a family history positive for potential genetic disorders.

Acknowledgments The support of Ymkje Cuperus in collecting the patient data is gratefully acknowledged. We thank Jackie Senior for critically reading the manuscript.

Open Access This article is distributed under the terms of the Creative Commons Attribution Noncommercial License which permits any noncommercial use, distribution, and reproduction in any medium, provided the original author(s) and source are credited. 


\section{References}

1. Devroey P, Van Steirteghem A. A review of ten years experience of ICSI. Hum Reprod Update. 2004;10:19-28. doi:10.1093/ humupd/dmh004.

2. Assisted reproductive technology in the United States. 1999 results generated from the american society for reproductive medicine/ society for assisted reproductive technology registry. Fertil Steril. 2002;78:918-31. doi:10.1016/S0015-0282(02)04198-5.

3. Andersen AN, Goossens V, Ferraretti AP, Bhattacharya S, Felberbaum R, de Mouzon J, et al. Assisted reproductive technology in Europe, 2004: results generated from European registers by ESHRE. Hum Reprod. 2008;23:756-71. doi:10.1093/ humrep/den014.

4. Kremer JA, Bots RS, Cohlen B, Crooij M, van Dop PA, Jansen CA, et al. Ten years of results of in-vitro fertilisation in the Netherlands 1996-2005. Ned Tijdschr Geneeskd. 2008;152:146-52.

5. Twisk M, Mastenbroek S, van Wely M, Heineman MJ, Van der Veen F, Repping S. Preimplantation genetic screening for abnormal number of chromosomes (aneuploidies) in in vitro fertilisation or intracytoplasmic sperm injection. Cochrane Database Syst Rev. 2006;(1):CD005291.

6. Friedler S, Raziel A, Strassburger D, Schachter M, Soffer Y, Ron-El R. Factors influencing the outcome of ICSI in patients with obstructive and non-obstructive azoospermia: a comparative study. Hum Reprod. 2002;17:3114-21. doi:10.1093/humrep/17.12.3114.

7. Steger K. Possible predictive factors for ICSI? Molecular biology techniques in combination with therapeutic testicular biopsies. Andrologia. 2003;35:200-8. doi:10.1046/j.1439-0272.2003. 00581.x.

8. Lewis $\mathrm{S}$, Klonoff-Cohen $\mathrm{H}$. What factors affect intracytoplasmic sperm injection outcomes? Obstet Gynecol Surv. 2005;60:111-23. doi:10.1097/01.ogx.0000153330.63877.9c.

9. Ferlin A, Arredi B, Foresta C. Genetic causes of male infertility. Reprod Toxicol. 2006;22:133-41. doi:10.1016/j.reprotox.2006. 04.016.

10. Lissens W, Liebaers I, Van Steirteghem A. Male infertility. In: Rimoin DL, Connor JM, editors. Emery and Rimoin's principles and practice of medical genetics. Churchill Livingstone; 2002. pp. 961-981.

11. World Health Organisation. WHO laboratory manual for the examination of human semen and sperm-cervical mucus interaction. 4th ed. Cambridge (UK): Cambridge University Press; 1999.

12. Bor P, Hindkjaer J, Kolvraa S, Ingerslev HJ. Y-chromosome microdeletions and cytogenetic findings in unselected ICSI candidates at a Danish fertility clinic. J Assist Reprod Genet. 2002;19:224-31. doi:10.1023/A:1015358802577.

13. Tuerlings JH, de France HF, Hamers A, Hordijk R, Van Hemel JO, Hansson K, et al. Chromosome studies in 1792 males prior to intra-cytoplasmic sperm injection: the Dutch experience. Eur J Hum Genet. 1998;6:194-200. doi:10.1038/sj.ejhg.5200193.

14. Vincent MC, Daudin M, De MP, Massat G, Mieusset R, Pontonnier F, et al. Cytogenetic investigations of infertile men with low sperm counts: a 25-year experience. J Androl. 2002;23:18-22. discussion 44-5.

15. Cruger DG, Agerholm I, Byriel L, Fedder J, Bruun-Petersen G. Genetic analysis of males from intracytoplasmic sperm injection couples. Clin Genet. 2003;64:198-203. doi:10.1034/j.13990004.2003.00128.x.

16. Mak V, Zielenski J, Tsui LC, Durie P, Zini A, Martin S, et al. Proportion of cystic fibrosis gene mutations not detected by routine testing in men with obstructive azoospermia. JAMA. 1999;281:2217-24. doi:10.1001/jama.281.23.2217.

17. Claustres M, Guittard C, Bozon D, Chevalier F, Verlingue C, Ferec C, et al. Spectrum of CFTR mutations in cystic fibrosis and in congenital absence of the vas deferens in France. Hum Mutat. 2000;16:143-56. doi:10.1002/1098-1004(200008)16:2<143:: AID-HUMU7>3.0.CO;2-J.

18. Giltay JC, Kastrop PM, Tuerlings JH, Kremer JA, Tiemessen CH, Gerssen-Schoorl KB, et al. Subfertile men with constitutive chromosome abnormalities do not necessarily refrain from intracytoplasmic sperm injection treatment: a follow-up study on 75 Dutch patients. Hum Reprod. 1999;14:318-20. doi:10.1093/humrep/14.2.318.

19. Nap AW, Van Golde RJ, Tuerlings JH, De Sutter P, Pieters MH, Giltay JC, et al. Reproductive decisions of men with microdeletions of the Y chromosome: the role of genetic counselling. Hum Reprod. 1999;14:2166-9. doi:10.1093/humrep/14.8.2166. 